\title{
CrystEngComm
}

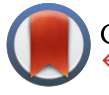

CrossMark click for updates

Cite this: CrystEngComm, 2016, 18 8871

Received 27th July 2016,

Accepted 19th October 2016

DOI: 10.1039/c6ce01652a

www.rsc.org/crystengcomm

\section{Structural transformations in crystals induced by radiation and pressure. Part 5 . The influence of pressure on the course of the photochemical reaction in crystals of 2,6-difluorocinnamic acid $\dagger$}

\author{
T. Galica, J. Bąkowicz, K. Konieczny and I. Turowska-Tyrk*
}

\begin{abstract}
The [2 + 2] photodimerization of 2,6-difluorocinnamic acid was gradually induced in crystals by UV radiation at $0.5,1.1$ and $2.1 \mathrm{GPa}$. The crystal and molecular structures were determined before the photochemical reaction and for many of its steps. For the pure monomer structures, the following parameters were analyzed in order to gain knowledge of the influence of pressure on the reaction environment: (a) the volume of free space, (b) the geometry of close intermolecular interactions, including hydrogen bonds and (c) the geometry of intermolecular reactivity parameters. The following structural changes brought about by the [2 + 2] photodimerization at high pressure were monitored step-by-step: (a) the cell parameters and the cell volume, (b) the content of the monomer and the dimer, (c) the distance between the reactive carbon atoms in adjacent monomer molecules and (d) the geometry of mutual orientation of molecules in the crystals. The rate of the [2+2] photodimerization of 2,6-difluorocinnamic acid increased as follows: $0.1 \mathrm{MPa}<0.5 \mathrm{GPa}<1.1 \mathrm{GPa} \leq 2.1 \mathrm{GPa}$. This was rationalized by the decrease in the volume of free space columns protecting the intermolecular geometry suitable for the reaction and by the decrease of the distance between the reactive carbon atoms of adjacent monomer molecules.
\end{abstract}

\section{Introduction}

Crystallographic studies under high pressure conditions are quite common nowadays, and many academic institutions and research centres around the world investigate the influence of pressure on crystal structures. However, the crystallographic experiments on monitoring photochemical reactions and structural changes caused by them at high pressure are still rare. ${ }^{1-4}$ Since pressure can influence the course of photochemical reactions and can change it in a different manner for different reactions, ${ }^{1-4}$ further research is certainly required.

The parameters describing the photochemical reactivity of molecules in crystals are well known, however, they are true only under atmospheric pressure. In the case of a $[2+2]$ photodimerization, the following geometrical parameters should be taken into account (their ideal values are given in the parentheses): ${ }^{5,6}$

$D$ - the distance between reacting molecules $(<4.2 \AA)$.

Faculty of Chemistry, Wroctaw University of Science and Technology, Wybrzeże Wyspiańskiego 27, 50-370 Wroctaw, Poland.

E-mail: ilona.turowska-tyrk@pwr.edu.pl; Fax: +48 713203364

$\dagger$ CCDC 1496295-1496307. For crystallographic data in CIF or other electronic format see DOI: 10.1039/c6ce01652a $\alpha$ - the $\mathrm{C}=\mathrm{C} \cdots \mathrm{C}$ angle between the double bonds of two adjacent molecules $\left(90^{\circ}\right)$.

$\kappa$ - the angle between the $>\mathrm{C}=\mathrm{C}<$ plane of one molecule and the plane of four reacting atoms $\left(90^{\circ}\right)$.

$\tau$ - the $\mathrm{C}=\mathrm{C} \cdots \mathrm{C}=\mathrm{C}$ torsion angle formed by the $\mathrm{C}=\mathrm{C}$ bonds of two adjacent molecules $\left(0^{\circ}\right)$.

$\phi$ - the angle between the $>\mathrm{C}=\mathrm{C}<$ fragments of two adjacent molecules $\left(0^{\circ}\right)$.

The ideal value of the above-given $D$ parameter ${ }^{7-9}$ can change at high pressure. Moreover, due to altered thermal vibrations and changes in molecular geometry, the real range of values at which a $[2+2]$ photodimerization happens can be different at high pressure.

Another important factor connecting mobility and geometry with molecular reactivity is associated with free space: its volume $\left(V_{\text {free }}\right)$, shape and elasticity. ${ }^{10-12}$ In general, since atomic movements are necessary for the reaction to occur, when the $V_{\text {free }}$ is too small the reaction may slow down or even halt. In particular, when the initial intermolecular geometry is far from ideal, but does not exclude the reaction, a bigger $V_{\text {free }}$ is desired. On the other hand, in the case of a geometry close to ideal, a small $V_{\text {free }}$ can be beneficial, since this protects the suitable intermolecular geometry during the phototransformation of crystals. The volume of free space, $V_{\text {free}}$, is correlated with the energy and force of the 
intermolecular interactions and thus with the intermolecular reactivity.

The next parameter important for the course of each reaction is the radiation wavelength connected with an absorption characteristic of a given compound. The wavelengths of light used to induce a photochemical reaction should correspond to a low energy absorption tail because this makes the photochemical reaction more uniform throughout the crystal. $^{13,14}$

The comparison of directions of structural changes brought about by a photochemical reaction conducted at ambient pressure with directions of structural deformations caused by high pressure itself can also help to rationalize molecular reactivity at high pressure. ${ }^{15,16}$

In this paper, we present the results of the research on structural transformations in crystals of 2,6-difluorocinnamic acid, compound $\mathbf{1}$, induced by UV radiation under conditions of high pressure and the comparison with the relevant data for ambient pressure. The equation of $[2+2]$ photodimerization for compound $\mathbf{1}$ is presented in Scheme 1. The results of the studies on the $[2+2]$ photodimerization of this compound under ambient conditions were presented previously. ${ }^{17}$ It is worth saying here that there are no publications concerning crystal structures of derivatives of cinnamic acid at high pressure, even for crystals containing only monomer molecules, i.e. before the photochemical reaction (CSD, ConQuest, version 1.18). ${ }^{18,19}$ However, the monitoring of structural changes brought about by the $[2+2]$ photodimerization in crystals under conditions of high pressure was carried out by crystallographic methods in the case of 2-benzyl-5benzylidenecyclopentanone. ${ }^{4}$

\section{Experimental}

2,6-Difluorocinnamic acid, compound 1, was purchased from Sigma-Aldrich. A diamond anvil cell (DAC) of the BoehlerAlmax type ${ }^{20}$ with a gasket made of inconel was used in high pressure experiments. The hydrostatic medium was a mixture of glycerin and water $(3: 2)$. The high pressure values were estimated by means of the unit cell parameters of quartz. ${ }^{21}$ Three crystals were used in the experiments at 0.5, 1.1 and $2.1 \mathrm{GPa}$, respectively. The first diffraction experiment for each crystal was conducted before UV irradiation. Afterwards, the crystals were irradiated inside the DAC by a set-up containing

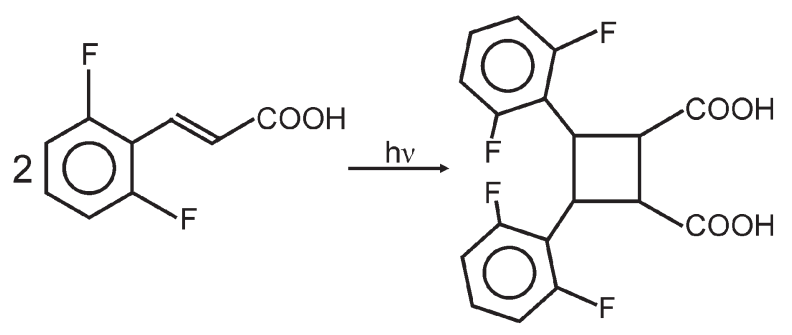

Scheme 1 The equation of the [2 + 2] photodimerization for 2,6difluorocinnamic acid. The molecules in the crystals are oriented head-to-head. a light source (a $100 \mathrm{~W}$ mercury lamp), a water filter and a WG-320 Andover optic glass filter. The WG-320 filter transmits $c a .100 \%$ radiation above $365 \mathrm{~nm}, 95 \%$ at $350 \mathrm{~nm}$ and $0 \%$ below $300 \mathrm{~nm}$, i.e. it transmits wavelengths corresponding to the low-energy absorption tail region of compound 1 . The selected glass filter made the studied photochemical reaction more uniform in the crystals. ${ }^{13,14}$ Both sides of the DAC containing the crystal inside were exposed to UV light repeatedly for $15 \mathrm{~s}$ with intervals of $10 \mathrm{~s}$. The UV irradiation experiments were carried out in the dark. In the case of crystal 1 at $0.5 \mathrm{GPa}$, the consecutive irradiation experiments were carried out for 60, 120 and $180 \mathrm{~s}$ in total. For crystal 2 at 1.1 $\mathrm{GPa}$, this was done every $30 \mathrm{~s}$. up to $270 \mathrm{~s}$ in total. Crystal 3 at $2.1 \mathrm{GPa}$ was irradiated in the following steps: 20, 50, 90, 120,180 and $270 \mathrm{~s}$ in total. The orientation of each irradiated crystal (thus the orientation of crystal faces) in the DAC was the same.

The gasket-shadow centering procedure was applied in order to align the DAC on a single-crystal X-ray diffractometer with a CCD detector. ${ }^{22}$ The data were collected under conditions of darkness. The data collection, UB matrices determination and data reduction were carried out using CrysAlis ${ }^{\text {Pro }}$ software. ${ }^{23}$ The corrections for the DAC absorption did not improve the quality of the data and were not applied. The $\mathrm{X}$-ray data sets contained reflections coming from compound 1, the quartz and two diamonds of the DAC. Nevertheless, it was possible to group them into four separated sets coming from each crystal and to use those coming from compound 1 during the subsequent structure determinations. Crystal 1 after 0 and $60 \mathrm{~s}$ of UV irradiation at $0.5 \mathrm{GPa}$ contained 100 and $88.8(8) \%$ of the monomer, respectively. Crystal 2 after 0,30 , 60 and $90 \mathrm{~s}$ of UV irradiation at $1.1 \mathrm{GPa}$ contained 100, 84.1(9), 81.1(11) and 81.2(10)\% of the monomer, respectively. Crystal 3 after $0,20,50,90,120,180$ and $270 \mathrm{~s}$ of UV irradiation at $2.1 \mathrm{GPa}$ contained 100, 89.0(7), 86.4(8), 81.2(9), $75.5(10), 71.4(11)$ and $67.6(12) \%$ of the monomer, respectively. The structures for the remaining times of UV irradiation were not of satisfactory quality.

The structures were refined with the use of SHELXL2014..$^{24,25}$ For the pure monomer crystals, there was no need to use restraints. For some atoms of the partiallyreacted crystals, the following restraints were used: DFIX - to keep an appropriate bond length between atoms, DANG - to keep an appropriate valence angle between atoms, FLAT - to ensure coplanarity of atoms and SIMU - so that selected atoms would have similar displacement parameters. The target values for commands DFIX and DANG were taken from the structures of the pure monomer and pure dimer crystals determined at $0.1 \mathrm{MPa} .{ }^{17}$

The initial atomic coordinates for the pure monomer crystals at $0.5,1.1$ and $2.1 \mathrm{GPa}$ were also taken from the structure determined at $0.1 \mathrm{MPa} .{ }^{17}$ Due to a small number of observed reflections $\left(F_{\mathrm{O}}>4 \sigma\left(F_{\mathrm{o}}\right)\right), \mathrm{F}$ and $\mathrm{O}$ atoms and a few $\mathrm{C}$ atoms (C2, C3 and C4 for 0.5 GPa; C3 and C9 for 1.1 and 2.1 GPa) were refined anisotropically and the remaining atoms isotropically. The crystals containing the monomer and dimer 
featured a disorder; nevertheless, all atoms from both molecules were separated over two positions: coming from the monomer and from the dimer. For these crystals, the 2,6difluorobenzene ring and the carboxyl group (with $\mathrm{H}$ atoms omitted) were refined as rigid rotating groups. The geometry of these fragments was taken from the structure of the monomer and the structure of the dimer determined at $0.1 \mathrm{MPa} .{ }^{17}$ For the crystal irradiated during 20 and $50 \mathrm{~s}$ at $2.1 \mathrm{GPa}$, the $\mathrm{F}$ and $\mathrm{O}$ atoms in monomer molecules were refined anisotropically. For the crystals irradiated during $60 \mathrm{~s}$ at $0.5 \mathrm{GPa}, 30 \mathrm{~s}$ at $1.1 \mathrm{GPa}$ and $90 \mathrm{~s}$ at $2.1 \mathrm{GPa}$, respectively, the $\mathrm{F}$ atoms in the monomer were refined anisotropically. For the remaining structures, all atoms were regarded as isotropic. The treatment of $\mathrm{F}$ and $\mathrm{O}$ atoms isotropically did not influence the calculated reaction yields in a significant manner. The mean difference between the reaction yields was 1.6(6)\%. Hydrogen atoms for all structures were treated geometrically with $U_{\text {iso }}$ equal to 1.2 or $1.5 U_{\text {eq. }}$.

The selected experimental and crystal data are presented in Table 1.

It is worth mentioning that the crystals of compound 1 undergoing the photochemical reaction under ambient conditions ${ }^{17}$ lost their quality quicker than the crystals undergoing such a reaction at higher pressure. Due to this, it was possible to monitor the structural changes brought about by the photochemical reaction at 1.1 and $2.1 \mathrm{GPa}$ within a broader range.

\section{Results and discussion}

Molecules of 2,6-difluorocinnamic acid, compound 1, in crystals are arranged in a head-to-head manner and form translational stacks along the $a$ axis both under ambient ${ }^{17}$ and high-pressure conditions. The high pressure significantly influences the geometry of the crystal lattice: it shortens the cell parameters, decreases free space and intermolecular distances and increases intermolecular interactions. It also influences weak intramolecular geometrical parameters as torsion angles. The above-mentioned items are related to each other.

The free space in the crystal of compound $\mathbf{1}$ is located mostly between the stacks of molecules. There is no significant free space between the molecules in one stack. Fig. 1 presents this characteristic for compound 1 at 0.5 and 2.1 $\mathrm{GPa}$. The free space in the unit cell of compound 1 changes from 69, through 51 and 38 to $24 \AA^{3}$ en route from $0.1 \mathrm{MPa}$ through $0.5 \mathrm{GPa}$ and $1.1 \mathrm{GPa}$ to $2.1 \mathrm{GPa}$, respectively i.e. by $65 \%$ in total. Such an exponential decrease is understandable since it is more and more difficult to move molecules closer to each other at higher pressure.

The change in the volume of the free space is accompanied by an $11 \%$ change in the volume of the unit cell, namely from 822.90(9) through 794.05(18) and 778.8(2) to 734.7(9) $\AA^{3}$ at 0.1 $\mathrm{MPa}, 0.5 \mathrm{GPa}, 1.1 \mathrm{GPa}$ and $2.1 \mathrm{GPa}$, respectively. The total percentage change is typical for crystals of molecular compounds. $^{1,27,28}$ The above-given variations in the cell vol- ume can be described by the Birch-Murnaghan equation of state: ${ }^{29}$

$$
P=3 / 2 K_{0}\left[\left(V_{0} / V\right)^{7 / 3}-\left(V_{0} / V\right)^{5 / 3}\right]\left\{1-3 / 4\left(4-K_{0}^{\prime}\right)\left[\left(V_{0} / V\right)^{2 / 3}-1\right]\right\},
$$

where $V_{0}$ is the cell volume at ambient pressure, $K_{0}$ is the ambient-pressure bulk modulus (incompressibility) and $K_{0}^{\prime}$ is the pressure derivative of incompressibility. For compound 1 , the least-squares fit gave $K_{0}=17.2 \mathrm{GPa}, K_{0}^{\prime}=1.6 \mathrm{GPa}$ and the correlation coefficient $r=0.991$.

In general, a small volume of a reaction cavity and free space makes movements of atoms and molecules more difficult and due to this, the reactivity of molecules can be reduced. $^{30-32}$ However, it is easy to assume a situation wherein the shape and extent of free space can influence a reaction in an opposite manner (see the Introduction section). Such an effect can take place in the case of compound 1. The free space in the crystal, similarly as molecules, also forms columns (see Fig. 1) and the decrease of its volume stiffen the stacks of molecules and this makes molecular movements more difficult. Since arising product molecules have a slightly different shape from a pair of monomer molecules, they force monomers to move slightly out of their initial positions. The thin regions of free space counteract such movements of monomers during the crystal phototransformation and help to retain the initial intermolecular geometry suitable for the photochemical reaction. The thinner are the columns of free space, the stronger is the effect. However, it is also possible that too high pressure can disable movements of atoms of monomers and in this way also halt the photochemical reaction. In the case when the shape and size of dimer molecules fit into a lattice of monomer pairs, a pressureinduced change in the volume of free space should not significantly influence a reaction. Such a situation was observed for 2-benzyl-5-benzylidenecyclopentanone. ${ }^{4}$ The abovedescribed changes in the volume of free space and the unit cell of compound 1 signify the decrease of distances between molecules and the increase of intermolecular interactions. Table 2 presents the intermolecular interactions shorter than the sum of van der Waals radii for $0.1 \mathrm{MPa}, 0.5 \mathrm{GPa}, 1.1 \mathrm{GPa}$ and $2.1 \mathrm{GPa}$ and Fig. 2 visualizes them for $2.1 \mathrm{GPa}$. As can be seen, the molecules are connected into dimers by $\mathrm{O}-\mathrm{H} \cdots \mathrm{O}$ bonds created by the carboxylic groups and further form planes of $\mathrm{C}-\mathrm{H} \cdots \mathrm{O}$ and $\mathrm{F} \cdots \mathrm{F}$ interactions. Along with the increase of pressure, these strong $\mathrm{O}-\mathrm{H} \cdots \mathrm{O}$ hydrogen bonds do not change their geometry, in contrast to the weaker C$\mathrm{H} \cdots \mathrm{O}$ and $\mathrm{F} \cdots \mathrm{F}$ interactions. Additionally, the distance between the phenyl rings drops below the sum of the van der Waals radii at $2.1 \mathrm{GPa}$. The two reacting adjacent molecules are situated in one stack, but they are elements of two different planes of intermolecular $\mathrm{O}-\mathrm{H} \cdots \mathrm{O}, \mathrm{C}-\mathrm{H} \cdots \mathrm{O}$ and $\mathrm{F} \cdots \mathrm{F}$ interactions. The increase of the intermolecular interactions, if they counteract movements of reactive atoms which is the case for compound 1 , should make the photochemical reaction more difficult. 
Table 1 The selected crystal and experimental data

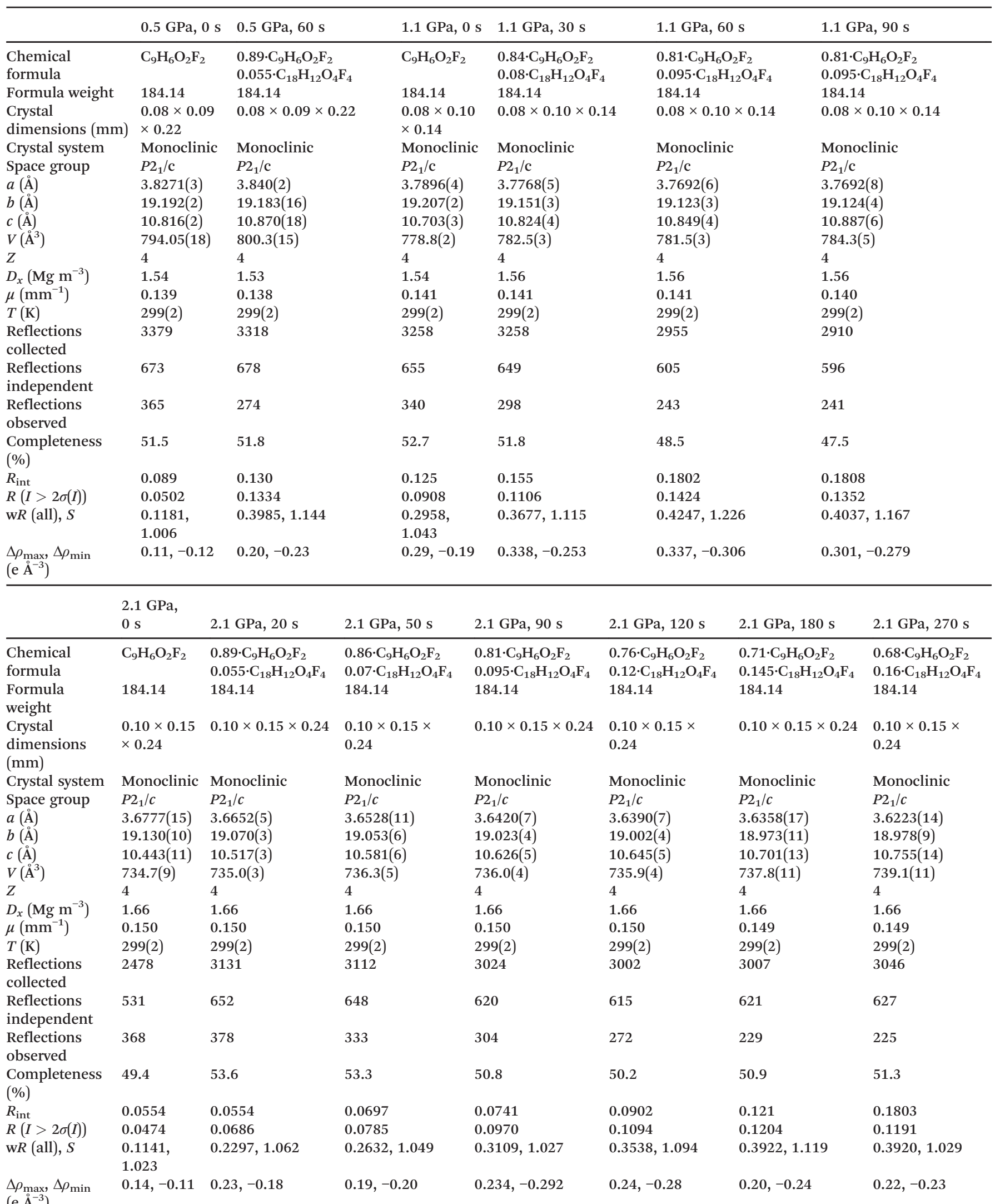
$\left(\mathrm{e} \AA^{-3}\right)$ 
(a)

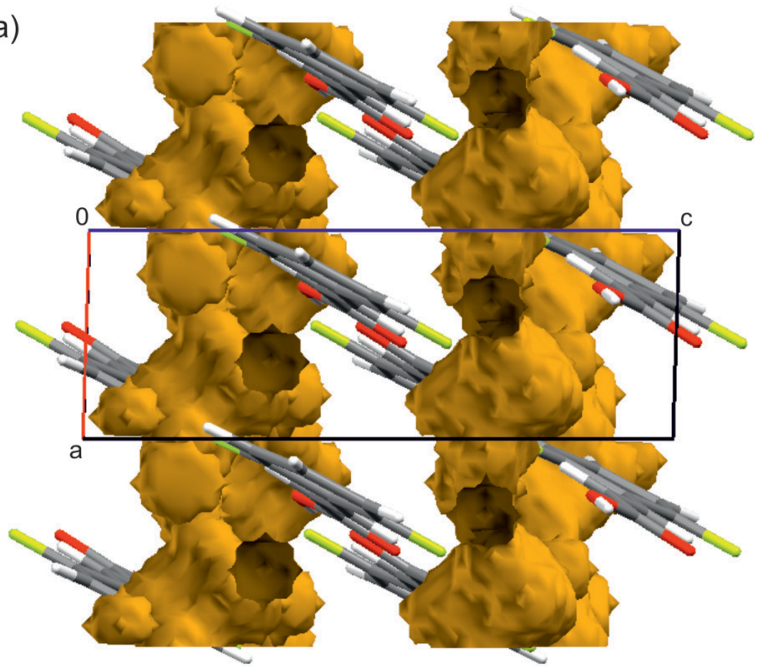

(b)

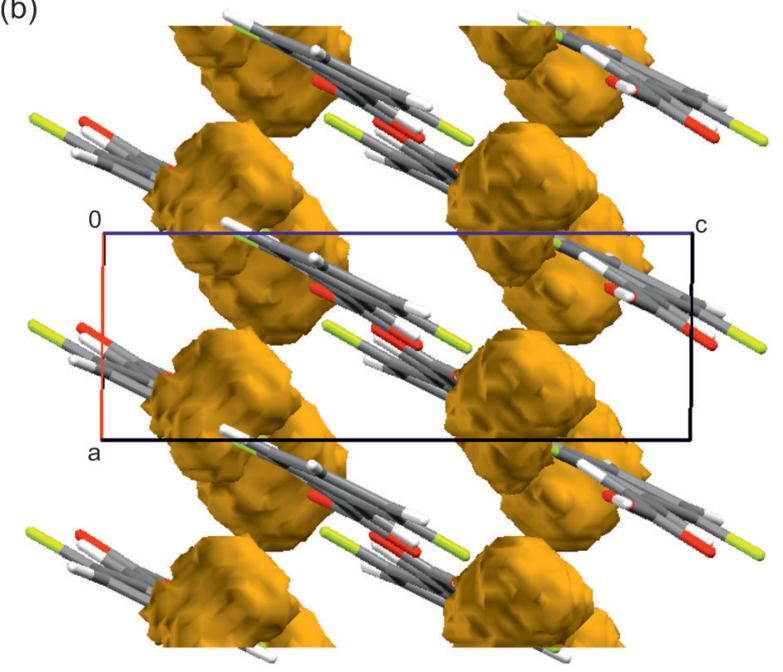

Fig. 1 The free space in the crystal at (a) $0.5 \mathrm{GPa}$ and (b) $2.1 \mathrm{GPa}$. The free spaces were calculated for the ball radius of $0.6 \AA$ and the grid of $0.2 \AA$ with the use of the Mercury program. ${ }^{26}$

The above-discussed changes in intermolecular geometry are accompanied by the decrease in the distance between the reactive atoms of two adjacent molecules in one stack, $D$, which is shown in Fig. 3a. The decrease in this distance should make the photochemical reaction easier.

High pressure influences not only intermolecular distances, but additionally modifies angular parameters of adjacent molecules. Fig. $3 \mathrm{~b}$ and $\mathrm{c}$ present the changes in the $\mathrm{C}=\mathrm{C} \cdots \mathrm{C}$ angle between the double bonds of two adjacent molecules and in the angle between the $>\mathrm{C}=\mathrm{C}<$ plane of one molecule and the plane of four reacting atoms (angles $\alpha$ and $\kappa$, see the Introduction section). High pressure also has a slight impact on the orientation of flat monomer molecules in the unit cell. Although the changes in the orientation along with pressure are small $\left(c a .1 .5^{\circ}\right)$, a smooth relationship exists. High pressure does not influence the internal geometry of molecules of compound 1: neither bond lengths, bond angles nor dihedral angles between rigid molecular fragments are affected.
Table 2 The geometry of intermolecular interactions in the crystal of compound $1[\AA \AA$, o]

\begin{tabular}{lllll}
\hline Pressure & $\mathrm{O} 1-\mathrm{H} 1$ & $\mathrm{H} 1 \cdots \mathrm{O} 2^{\mathrm{i}}$ & $\mathrm{O} 1 \cdots \mathrm{O} 2^{\mathrm{i}}$ & $\mathrm{O} 1-\mathrm{H} 1 \cdots \mathrm{O} 2^{\mathrm{i}}$ \\
\hline $0.1 \mathrm{MPa}$ & $0.93(3)$ & $1.73(3)$ & $2.666(2)$ & $176(2)$ \\
$0.5 \mathrm{GPa}$ & 0.82 & 1.84 & $2.661(4)$ & 177 \\
$1.1 \mathrm{GPa}$ & 0.82 & 1.84 & $2.658(10)$ & 177 \\
$2.1 \mathrm{GPa}$ & 0.82 & 1.84 & $2.657(5)$ & 174 \\
\hline Pressure & $\mathrm{C} 2-\mathrm{H} 2$ & $\mathrm{H} 2 \cdots \mathrm{O} 2^{\mathrm{ii}}$ & $\mathrm{C} 2 \cdots \mathrm{O} 2^{\mathrm{ii}}$ & $\mathrm{C} 2-\mathrm{H} 2 \cdots \mathrm{O} 2^{\mathrm{ii}}$ \\
\hline $0.1 \mathrm{MPa}$ & $0.90(2)$ & $2.54(2)$ & $3.427(3)$ & $169.1(16)$ \\
$0.5 \mathrm{GPa}$ & 0.93 & 2.48 & $3.385(8)$ & 166 \\
$1.1 \mathrm{GPa}$ & 0.93 & 2.47 & $3.388(16)$ & 169 \\
$2.1 \mathrm{GPa}$ & 0.93 & 2.38 & $3.289(8)$ & 166 \\
\hline Pressure & \multicolumn{2}{c}{$\mathrm{F} 1 \cdots \mathrm{F} 2^{\mathrm{ii}}$} & $\pi \cdots \pi^{\mathrm{iii}}$ & \\
\hline $0.1 \mathrm{MPa}$ & $2.807(16)$ & 3.886 & \\
$0.5 \mathrm{GPa}$ & $2.756(4)$ & 3.827 & \\
$1.1 \mathrm{GPa}$ & $2.723(8)$ & 3.790 & \\
$2.1 \mathrm{GPa}$ & $2.652(4)$ & 3.678 &
\end{tabular}

Symmetry codes for the $0.1 \mathrm{MPa}$ structure: $\mathrm{i}=2-x, 2-y, 1-z$; ii $=$ $-1+x, 3 / 2-y,-1 / 2+z$; iii $=x+1, y, z$. Symmetry codes for the high pressure structures: $\mathrm{i}=1-x, 2-y,-z$; ii $=1+x, 3 / 2-y, 1 / 2+z$; iii $=$ $x+1, y, z$.

There is also another factor which can have an impact on the reaction path and the reaction rate. Namely, if a given reaction proceeds with an increase of volume of a unit cell under ambient conditions, then imposing high pressure should make its proceeding more difficult. However, a few exceptions from this rule were also encountered. ${ }^{15,16}$ In the case of compound 1 under ambient conditions, the cell volume increases at the beginning of the crystal phototransformation, ${ }^{17}$ which should decrease the reaction rate at high pressure. We described above the features of the crystal structures before UV irradiation to present the initial reaction environment and to show how pressure modifies it before the reaction. The photochemical reactivity of molecules in the crystal of compound $\mathbf{1}$ is a result of the simultaneous influence of the above-described factors.

The course of the photochemical reaction of compound 1 under various values of high pressure was monitored by

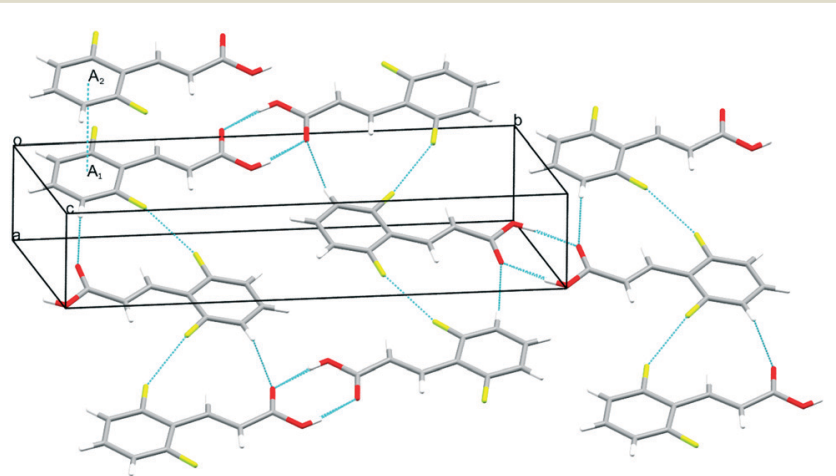

Fig. 2 The plane of intermolecular interactions shorter than the sum of van der Waals radii in the crystal at $2.1 \mathrm{GPa}$, prepared with the Mercury program. ${ }^{26}$ Molecules $A_{1}$ and $A_{2}$ are situated in one stack, but they form two separated planes of interactions. 
(a)

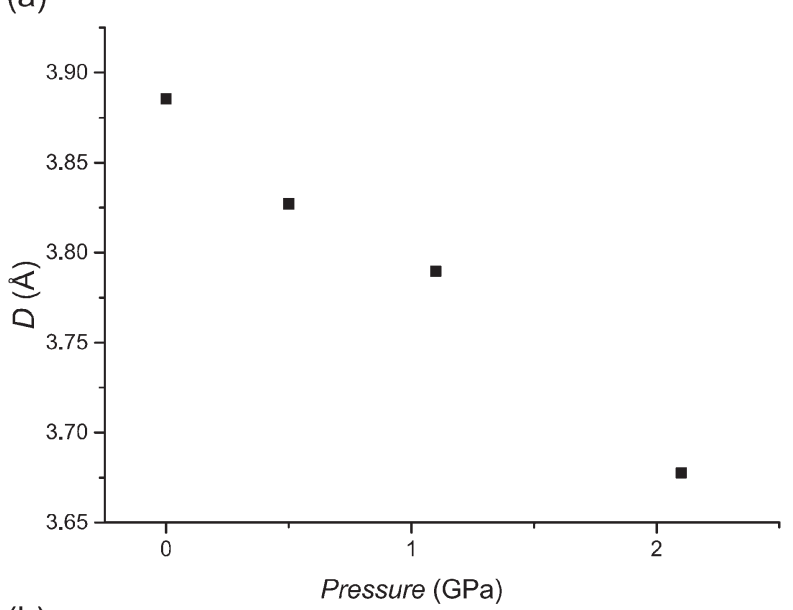

(b)

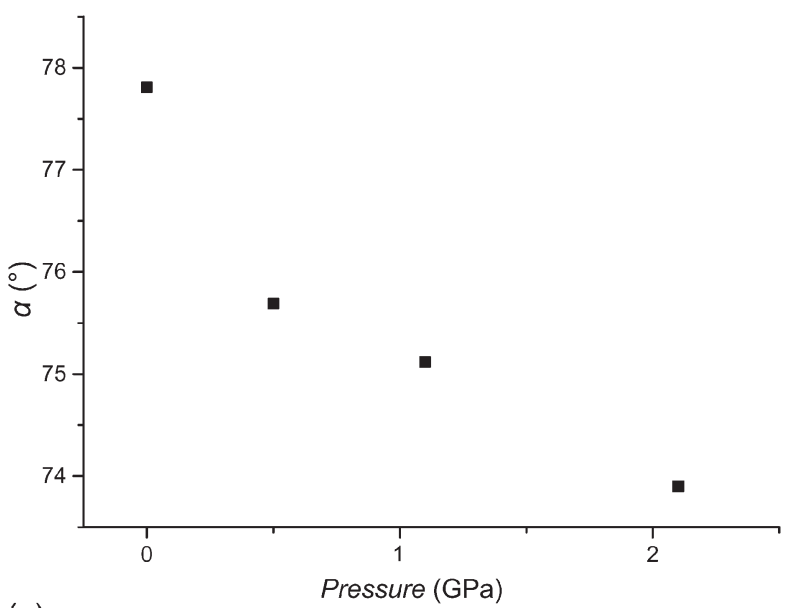

(c)

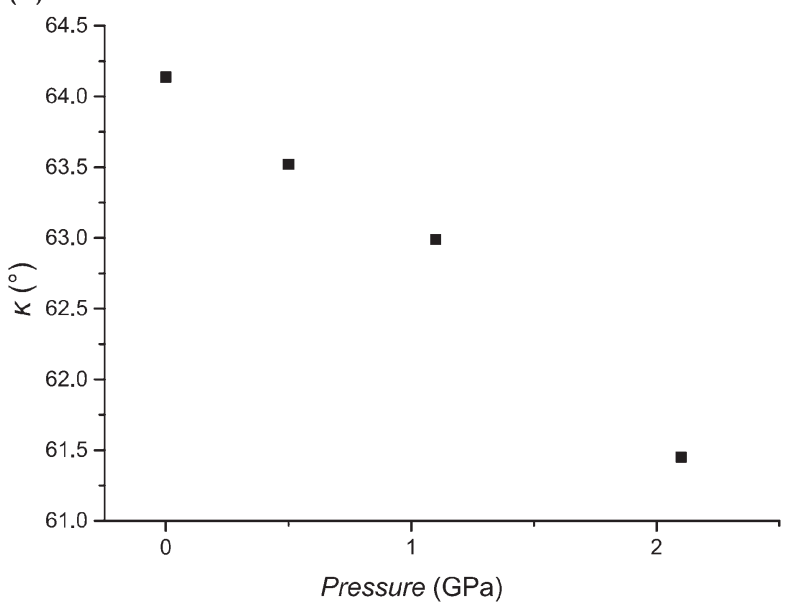

Fig. 3 The variations in (a) the distance between reactive carbon atoms of two adjacent monomer molecules, (b) the angle between the double bonds of two adjacent monomer molecules and (c) the angle between the $>\mathrm{C}=\mathrm{C}<$ plane of one monomer molecule and the plane of four reacting atoms along with the increase of pressure. The standard deviations for $D, \alpha$ and $\kappa$ were in the ranges of $0.0003-$ $0.0015 \AA$, $0.1-0.4^{\circ}$ and $0.2-0.6^{\circ}$, respectively.

means of the unit cell parameters, the content of the monomer (and the dimer) in the crystals, the intermolecular geo- metrical parameters as the distance between the reactive carbon atoms and by the orientation of monomer molecules. The variations in the cell parameters along with the time of UV irradiation of the crystals at $0.5,1.1$ and $2.1 \mathrm{GPa}$ are shown in Fig. 4. As can be seen, the presented relationships are smooth. At $0.5 \mathrm{GPa}$, cell parameter $a$ slightly increases at the beginning and afterwards decreases; parameter $b$ after a certain reduction increases; $c$ increases and the cell volume after a certain increase starts to decrease. The same character of changes was observed for the reaction conducted under ambient conditions. ${ }^{17}$

The further increase of pressure altered the character of the above-described changes, namely, at 1.1 and $2.1 \mathrm{GPa}$, cell parameters $a$ and $b$ decrease while $c$ increases. The biggest variations are at the beginning of the photochemical transformation. The decrease in parameter $a$ can be rationalized by the fact that one dimer molecule occupies less space along the $a$ axis than one pair of monomer molecules, which is visualized in Fig. 5. The extent of the changes in parameter $b$ along with the reaction progress is very similar for 1.1 and $2.1 \mathrm{GPa}$. The same is true for parameter $c$. In contrast, the decrease in parameter $a$ is statistically bigger in 2.1 than in 1.1 GPa.

The variations in the values of parameters $a, b$ and $c$ are greater for 1.1 and $2.1 \mathrm{GPa}$ than for $0.5 \mathrm{GPa}$. For $0.5 \mathrm{GPa}$, parameters $a, b$ and $c$ were altered after $120 \mathrm{~s}$ by 0.0127 , 0.039 and $0.085 \AA$ i.e. by $0.33,0.20$ and $0.78 \%$, respectively. For 1.1 GPa the respective changes for the same time were $0.0207,0.077$ and $0.149 \AA$, i.e. $0.55,0.40$ and $1.40 \%$ and for $2.1 \mathrm{GPa}$ they were $0.0249,0.077$, and $0.138 \AA$, i.e. $0.68,0.40$ and $1.32 \%$, respectively. Moreover, the reactant content in the crystals after $90 \mathrm{~s}$ of UV irradiation is the same for 1.1 and $2.1 \mathrm{GPa}: 81.2(10) \%$ and $81.2(9) \%$, respectively. In the case of $0.1 \mathrm{MPa}$, the changes in the cell parameters were slower. ${ }^{17}$

On the grounds of the above data, the trend of the changes in the reaction rate of compound 1 can be presented as follows: $0.1 \mathrm{MPa}<0.5 \mathrm{GPa}<1.1 \mathrm{GPa} \leq 2.1 \mathrm{GPa}$. A similar trend was also noticed for 2-benzyl-5-benzylidenecyclopentanone, namely, the reaction rate was higher at $0.7 \mathrm{GPa}$ than $0.5 \mathrm{GPa}^{4}$

The variations in the reactant content along with the time of UV irradiation at $2.1 \mathrm{GPa}$ are shown in Fig. 6. The relationship has an exponential character, i.e. variations are quicker at the beginning of the photochemical crystal transformation. The exponential character of such relationships was also observed for the $[2+2]$ photodimerization of 2-benzyl-5benzylidenecyclopentanone studied at ambient ${ }^{34,36}$ and high $^{4}$ pressures and 2-benzylidene-5-(4-chlorobenzyl)cyclopentanone studied under ambient conditions. ${ }^{35,36}$

As written above, the $[2+2]$ photodimerization of compound 1 undergoes between two adjacent molecules situated in one translational stack. Since molecules react in a random way, it is possible that after a certain time of the $[2+2]$ photodimerization, a portion of the monomer molecules will not have a partner for the reaction. In the most extreme 

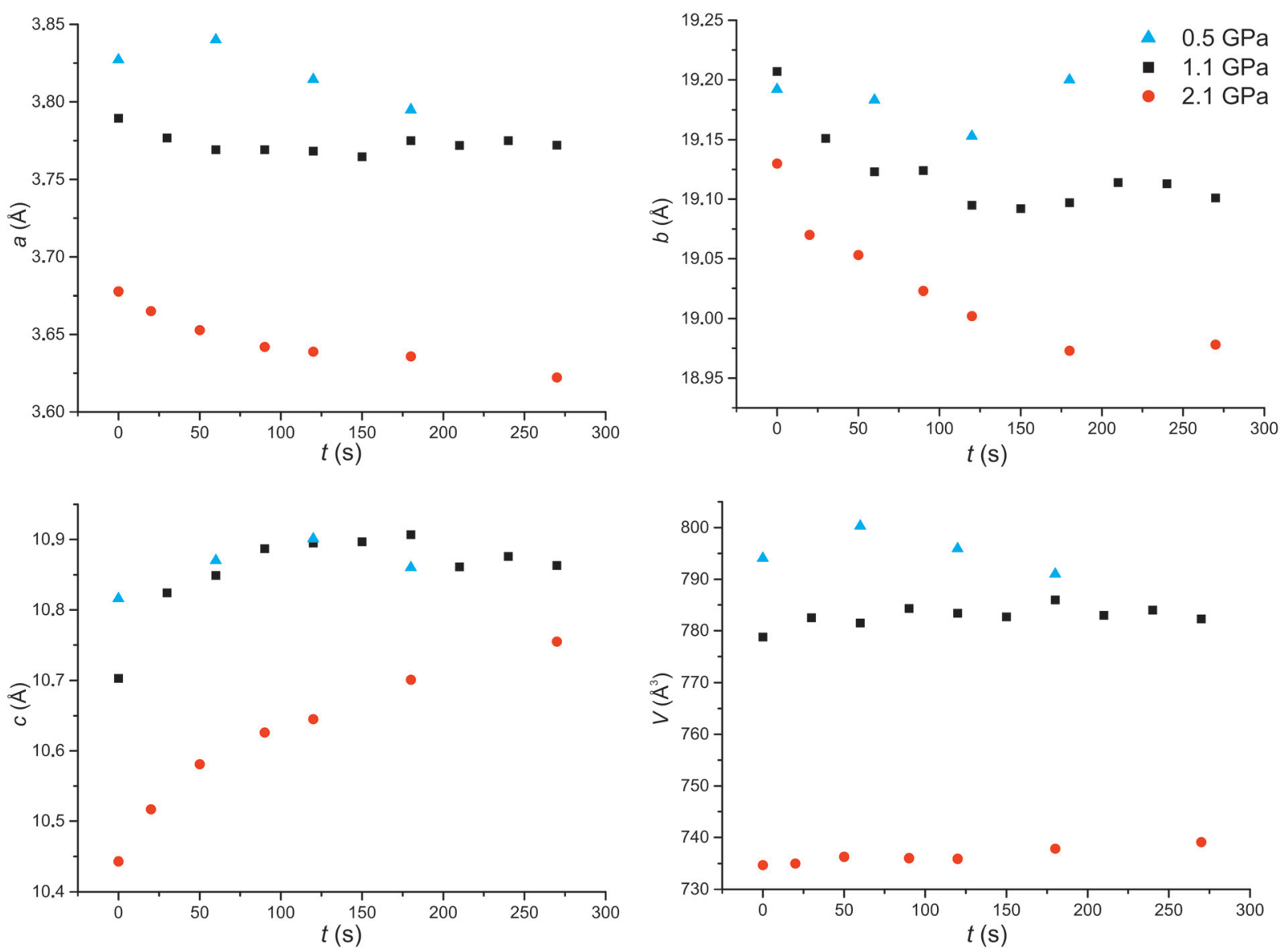

Fig. 4 The changes in the unit cell parameters along with the reaction progress.

situation, one third of the monomer molecules may remain unreacted.

The next monitored parameter is the $D$ distance between the reactive carbon atoms in adjacent molecules. As the photochemical reaction of compound 1 progressed, the $D$ distance decreased in a linear way. The variations with the percentage decrease of the content of the monomer in the crystal at 1.1 and $2.1 \mathrm{GPa}$ are presented in Fig. 7. The linear character of such changes was also observed at ambient pressure for other compounds undergoing the $[2+2]$ photodimerization, namely 2-benzyl-5-benzylidenecyclopentanone, ${ }^{34,36}$ 2-benzylidene-5-(4-chlorobenzyl)cyclopentanone ${ }^{35,36}$ and 2-ethoxycinnamic acid, ${ }^{36,37}$ and it was explained by the stress imposed on reactant molecules by smaller product molecules: the more product, i.e. the less reactant, the stronger the effect.

Nevertheless, at high pressure 2-benzyl-5benzylidenecyclopentanone showed a different characteristic of such a relationship, however, at the beginning of the photochemical transformation the $D$ distance also decreased. ${ }^{4}$

As the photochemical reaction of compound 1 progressed, the variations in the orientation of monomer molecules proceeded. Although the biggest alteration in the angle between the molecular plane and the unit cell faces is small (ca. $3^{\circ}$ for the $X Y$ plane), the course of the relationship is smooth. Similar changes in orientation of molecules and rigid molecular fragments were also observed in the case of 2-benzyl-5-benzylidenecyclopentanone at high pressure. ${ }^{4}$ Changes were also noticed for other compounds undergoing $[2+2]$ photodimerization, but under ambient conditions, and in some cases, they were bigger. ${ }^{34-37}$

\section{Conclusions}

We presented the results on monitoring structural changes in crystals of 2,6-difluorocinnamic acid brought about by (a) high pressure itself and (b) the photochemical reaction conducted under high pressure. With the aim of achieving these results, we determined the high-pressure structures for the pure monomer crystals and for the partially reacted crystals, i.e. containing both the monomer and the dimer. The experiments were carried out at 0.5, 1.1 and $2.1 \mathrm{GPa}$. We discussed the influence of pressure on (a) the volume of free space, (b) the geometry of close intermolecular interactions, including hydrogen bonds, (c) the geometry of intermolecular reactivity parameters and (d) the mutual orientation of monomer molecules. We stated that (a) the decrease in the volume of free space columns and (b) the decrease in the distance between the reactive carbon atoms in adjacent monomer molecules, $D$, at high pressure can increase the rate of the $[2+2]$ 


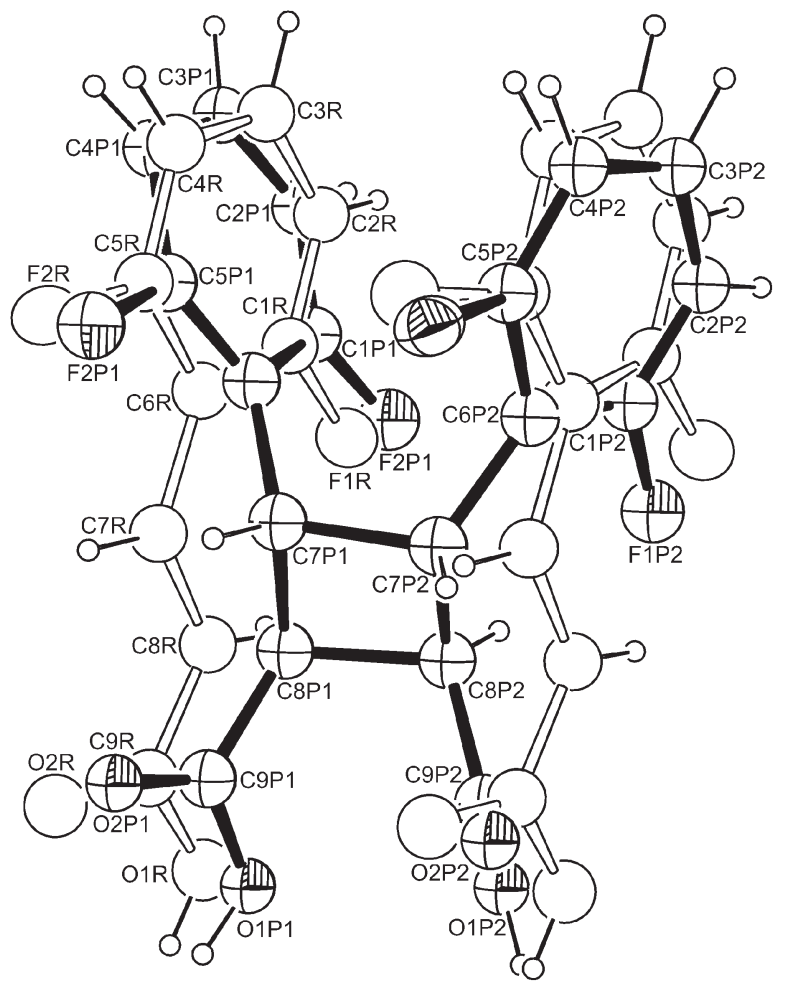

Fig. 5 A dimer molecule (solid bonds) and a pair of monomer molecules (empty bonds) in the crystal containing $81.2(9) \%$ of the monomer after $90 \mathrm{~s}$ of UV irradiation at $2.1 \mathrm{GPa}$, prepared with the Ortep program. ${ }^{33}$ The displacement ellipsoids were drawn at a $20 \%$ probability level. The asymmetric unit contains one monomer molecule and one dimer molecule.

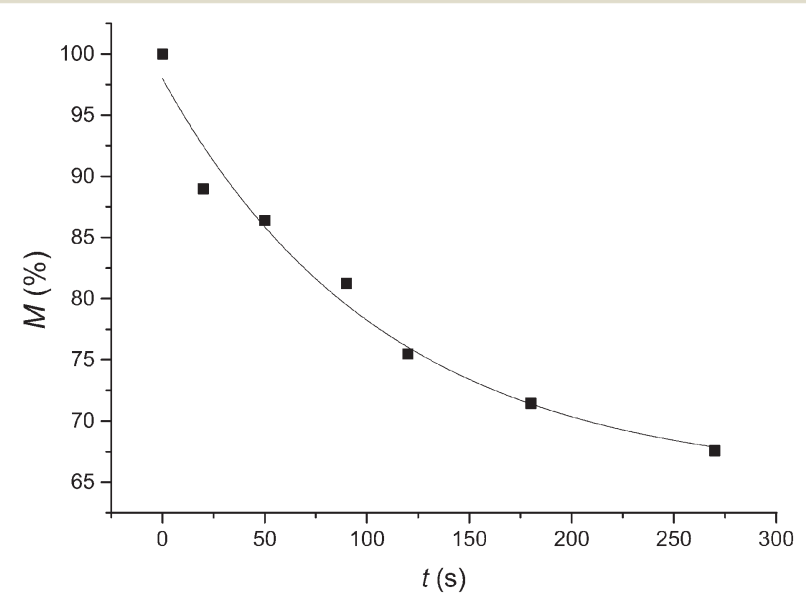

Fig. 6 The change in the monomer content along with the time of UV irradiation at $2.1 \mathrm{GPa}$. The equation of the fitted curve is $M=33 \exp (-t /$ 109) +65 and the correlation coefficient is $r=0.980$.

photodimerization of 2,6-difluorocinnamic acid. This increase was not reversed by the opposing factors such as (a) the increase of strength of intermolecular interactions forming planes, (b) the decrease of values of angular reactivity parameters $\alpha$ and $\kappa$ and (c) the initial increase of the unit cell volume with the reaction progress at ambient pressure. The rate of the $[2+2]$ photodimerization of 2,6-difluorocinnamic acid changed in the direction: $0.1 \mathrm{MPa}<0.5 \mathrm{GPa}<1.1 \mathrm{GPa} \leq 2.1 \mathrm{GPa}$.

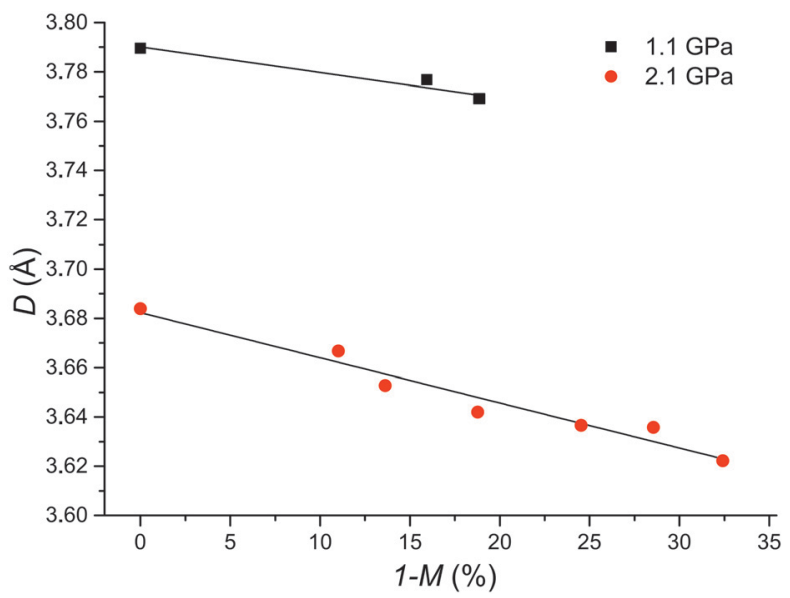

Fig. 7 The variations in the distance between the reactive atoms in two adjacent monomer molecules, $D$, along with the percentage decrease of the content of the monomer molecules in the crystal at 1.1 and $2.1 \mathrm{GPa}$.

The following structural changes brought about by the [2 $+2]$ photodimerization at high pressure were monitored stepby-step: (a) the cell parameters and the cell volume, (b) the content of the product in the crystal, (c) the distance between the reactive carbon atoms in adjacent monomer molecules and (d) the geometry of mutual orientation of molecules in the crystals. The character and the size of some structural changes were different for varying pressures.

In the scientific literature, there is only one publication on structural changes brought about by an intermolecular photochemical reaction conducted at high pressure and monitored by crystallographic methods. ${ }^{4}$ In our opinion, the studies on the course of [2+2] photodimerization of other compounds are needed, including also compounds not having a stacking architecture, in order to gain a broader knowledge of the reasons for the behaviour of molecules and crystalline materials at high pressure during this type of reaction.

\section{Acknowledgements}

This work was financed by the statutory activity subsidy of the Polish Ministry of Science and Higher Education.

\section{References}

1 J. Bąkowicz and I. Turowska-Tyrk, CrystEngComm, 2014, 16, 6039-6048.

2 K. Konieczny, J. Bąkowicz and I. Turowska-Tyrk, CrystEngComm, 2015, 17, 7693-7701.

3 K. Konieczny, J. Bąkowicz and I. Turowska-Tyrk, J. Photochem. Photobiol., A, 2016, 325, 111-115.

4 J. Bąkowicz and I. Turowska-Tyrk, CrystEngComm, 2016, DOI: 10.1039/c6ce01650b.

5 K. Gnanaguru, N. Ramasubbu, K. Venkatesan and V. Ramamurthy, J. Org. Chem., 1985, 50, 2337-2346.

6 I. Turowska-Tyrk and K. Grześniak, Acta Crystallogr., Sect. C: Cryst. Struct. Commun., 2004, 60, o146-0148.

7 G. M. J. Schmidt, J. Chem. Soc., 1964, 2014-2021. 
8 G. M. J. Schmidt and M. D. Cohen, J. Chem. Soc., 1964, 1996-2000.

9 G. M. J. Schmidt, Pure Appl. Chem., 1971, 27, 647-678.

10 E. V. Boldyreva, Solid State Ionics, 1997, 101-103, 843-849.

11 E. V. Boldyreva, Russ. J. Coord. Chem., 2001, 27, 297-323.

12 M. Tapilin, N. N. Bulgakov, A. P. Chupakhin, A. A. Politov and A. G. Druganov, J. Struct. Chem., 2010, 51, 635-641.

13 V. Enkelmann, G. Wegner, K. Novak and K. B. Wagener, J. Am. Chem. Soc., 1993, 115, 10390-10391.

14 K. Novak, V. Enkelmann, G. Wegner and K. B. Wagener, Angew. Chem., Int. Ed. Engl., 1993, 32, 1614-1616.

15 E. V. Boldyreva, S. L. Kuzmina and H. Ahsbahs, J. Struct. Chem., 1998, 39, 762-773.

16 B. A. Zakharov, A. S. Marchuk and E. V. Boldyreva, CrystEngComm, 2015, 17, 8812-8816.

17 T. Galica, J. Backowicz, P. Broda and I. Turowska-Tyrk, Acta Crystallogr., Sect. C: Struct. Chem., 2016, 72, 593-599.

18 I. J. Bruno, J. C. Cole, P. R. Edgington, M. Kessler, C. F. Macrae, P. McCabe, J. Pearson and R. Taylor, Acta Crystallogr., Sect. B: Struct. Sci., 2002, 58, 389-397.

19 C. R. Groom and F. H. Allen, Angew. Chem., Int. Ed., 2014, 53, 662-671.

20 R. Boehler, Rev. Sci. Instrum., 2006, 77, 115103.

21 R. J. Angel, D. R. Allan, R. Miletich and W. Finger, J. Appl. Crystallogr., 1997, 30, 461-466.

22 A. Budzianowski and A. Katrusiak in High-Pressure Crystallography, ed. A. Katrusiak and P. F. McMillan, Kluwer Academic Publishers, Dordrecht Boston London, 2004, pp. 101-112.
23 Agilent, CrysAlis $^{P R O}$, Agilent Technologies, Wrocław, Poland, 2014.

24 G. M. Sheldrick, Acta Crystallogr., Sect. A: Found. Crystallogr., 2008, 64, 112-122.

25 G. M. Sheldrick, Acta Crystallogr., Sect. C: Struct. Chem., 2015, 71, 3-8.

26 C. F. Macrae, P. R. Edgington, P. McCabe, E. Pidcock, G. P. Shields, R. Taylor, M. Towler and J. van de Streek, J. Appl. Crystallogr., 2006, 39, 453-457.

27 A. Budzianowski, A. Olejniczak and A. Katrusiak, Acta Crystallogr., Sect. B: Struct. Sci., 2006, 62, 1078-1089.

28 E. V. Boldyreva, T. N. Drebushchak, T. P. Shakhtshneider, H. Sowa, H. Ahsbahs, S. V. Goryainov, S. N. Ivashevskaya, E. N. Kolesnik, V. A. Drebushchak and E. B. Burgina, ARKIVOC XII, 2004, pp. 128-155.

29 F. Birch, Phys. Rev., 1947, 71, 809-824.

30 J. N. Moorthy, P. Venkatakrishnan, G. Savitha and R. G. Weiss, Photochem. Photobiol. Sci., 2006, 5, 903-913.

31 I. Zouev, T. Lavy and M. Kaftory, Eur. J. Org. Chem., 2006, 4164-4169.

32 Y. Ito, H. Takahashi, J. Hasegawa and N. J. Turro, Tetrahedron, 2009, 65, 677-689.

33 L. J. Farrugia, J. Appl. Crystallogr., 2012, 45, 849-854.

34 I. Turowska-Tyrk, Chem. - Eur. J., 2001, 7, 3401-3405.

35 I. Turowska-Tyrk, Acta Crystallogr., Sect. B: Struct. Sci., 2003, 59, 670-675.

36 I. Turowska-Tyrk, J. Phys. Org. Chem., 2004, 17, 837-847.

37 M. A. Fernandes and D. C. Levendis, Acta Crystallogr., Sect. B: Struct. Sci., 2004, 60, 315-324. 